\section{Mujeres trabajadoras con dolor crónico osteomuscular: análisis de caso}

\author{
Working women with chronic musculoskeletal pain: \\ a case series
}

\author{
Mulheres que trabalham com dor musculoesquelética \\ crônica: análise de caso
}

\author{
1 Centro Universitario \\ de Ciencias de la Salud, \\ Universidad de Guadalajara, \\ Guadalajara, México. \\ Correspondencia \\ C. A. Ordónez-Hernández \\ Centro Universitario de \\ Ciencias de la Salud, \\ Universidad de Guadalajara. \\ Centro Universitario de \\ Ciencias de la Salud, \\ Puerta 1, Edificio N, Planta \\ Alta, Sierra Mojada 950 C \\ Guadalajara/Jalisco - 44350, \\ México.
}

andrea-ordonez@hotmail.com

\begin{abstract}
This study aimed to analyze the experience of working women suffering from chronic musculoskeletal pain, using a qualitative design with a phenomenological approach. The technique drew on in-depth interviews with five working women that presented to the orthopedics and neurosurgery departments of a hospital in Guadalajara, Mexico, with a complaint of musculoskeletal pain for more than six months. The study showed that the women felt rejection, segregation, discrimination, lack of support at the workplace, and feelings of frustration and powerlessness related to their health condition. The women also perceived as a barrier the lack of efficiency in disability proceedings and job reintegration or relocation. Financial and family responsibilities were their main reason for continuing to work despite their chronic musculoskeletal pain.
\end{abstract}

Musculoskeletal Pain; Chronic Pain; Working Women; Women's Health
Cecilia Andrea Ordóñez-Hernández 1 Mónica Isabel Contreras-Estrada 1 Ruben Soltero-Avelar 1

\section{Resumen}

El objetivo de este estudio fue conocer las experiencias de mujeres trabajadoras con dolor crónico osteomuscular. El diseño fue cualitativo con enfoque fenomenológico. La técnica utilizada fue entrevista en profundidad, realizada a cinco mujeres trabajadoras que consultaron por dolor osteomuscular mayor a 6 meses en los servicios de ortopedia y neurocirugía de un hospital en Guadalajara, México. Se encontró percepción de rechazo, segregación, discriminación y falta de apoyo en su ambiente laboral a causa de su dolor, y sentimientos de frustración e impotencia relacionados con su padecimiento de salud. Se percibe como barrera la mala percepción del personal de la salud y la falta de eficiencia en los procesos de incapacidad, reintegración y reubicación laboral. Las responsabilidades económicas y familiares son la principal motivación para continuar trabajando con la presencia de dolor osteomuscular crónico.

Dolor Musculoesquelético; Dolor Crónico; Trabajo de Mujeres; Salud de la Mujer 


\section{Introducción}

Las mujeres trabajadoras que laboran con el padecimiento de dolor crónico osteomuscular deben cumplir con las tareas y responsabilidades que tienen asignadas, con una productividad esperable que no contempla en ningún caso la presencia de la enfermedad. Por su etiología, los desórdenes osteomusculares, son altamente incapacitantes, limitan la funcionalidad de los segmentos corporales e impiden algunas de las habilidades imprescindibles para trabajar como la movilidad, el desplazamiento y la manipulación de cargas 1 .

Los desórdenes osteomusculares requieren en su mayoría de períodos de resolución prolongados, los cuales pueden llegar a convertirse en síndromes independientes de dolor crónico 2. Para algunas trabajadoras este tipo de dolor es generador de fuerte afección psicopatológica, ansiedad, depresión, estrés, ira y agresividad, que repercuten no sólo en el desempeño cotidiano de sus roles, sino que también afecta el tratamiento terapéutico haciéndolo poco efectivo o fallido 3,4 . La discapacidad temporal o definitiva para la realización de algunas tareas laborales genera inconformidad de la gerencia, de los compañeros de trabajo y en algunos casos inclusive en los clientes externos, lo cual podría dar lugar al despido o a la renuncia de la trabajadora. Las trabajadoras con dolor crónico osteomuscular se ven enfrentadas al trabajo, a la vida social y familiar en condiciones adversas y deben esforzarse para cumplir con las expectativas que se tiene de ellas en cada rol particular.

En este estudio se eligió el modelo de investigación cualitativa desde el enfoque fenomenológico, definido por Husserl 5 como método descriptivo fundado en la intuición y la esencia de las vivencias. Esta teoría se cuestiona por la verdadera naturaleza de los fenómenos a través de la descripción de los significados vividos y existenciales y busca explicar el significado de la realidad vivida, en un entorno particular.

Para lograr un acercamiento a la comprensión de las experiencias y vivencias de las mujeres trabajadoras con dolor crónico osteomuscular se utilizó la Sociología de la Vida Cotidiana de Berger \& Luckmann ${ }^{6}$ quienes consideran el análisis fenomenológico como el más conveniente para clarificar, describir, analizar e interpretar las experiencias vividas que caracterizan la vida cotidiana, la cual se presenta como una realidad interpretada por los hombres y que para ellos tiene el significado subjetivo de un mundo coherente.

Para estos autores, la realidad de la vida cotidiana se organiza alrededor del "aquí" del cuerpo y el "ahora" del presente, lo que constituye el realissimun de la conciencia, sin embargo no se agota por las presencias inmediatas, sino que abarca fenómenos que no están presentes, en diferentes grados de proximidad y alejamiento tanto espacial, como temporal. De esta manera, en esta investigación se retoman los cuatro componentes fenomenológicos básicos: el cuerpo, las relaciones humanas, el espacio y el tiempo 7, descritos a través de la realidad vivida, la interacción social, el lenguaje y el conocimiento, tanto en los sucesos rutinarios, como problemáticos de las mujeres que trabajan con el padecimiento de un dolor crónico.

\section{Método}

Se utilizó un diseño cualitativo de carácter fenomenológico ${ }^{8}$. El objetivo de este estudio de caso fue documentar la experiencia de mujeres trabajadoras que padecen dolor crónico osteomuscular, intentando comprender esta problemática a partir de la descripción y análisis de su propio contexto ${ }^{9}$. El estudio se realizó en un hospital de Guadalajara, Estado de Jalisco, México, que presta servicios de salud a los trabajadores del estado. Se aplicó el cuestionario de aceptación y catastrofización del dolor crónico a 88 mujeres activas laboralmente, institucionalizadas (empleadas dependientes de alguna institución pública del Estado de Jalisco), con dolor crónico osteomuscular superior a 6 meses, que no estaban calificadas en estado de invalidez y que desearon participar voluntariamente en la investigación. La aplicación de los instrumentos permitió el acercamiento cara a cara y la manifestación espontánea de las mujeres acerca de su problemática.

A través de este primer contacto fue posible seleccionar los casos, utilizando un muestreo intencional teórico ${ }^{10}$, se eligió a las informantes teniendo en cuenta la cronicidad de su dolor, el grado de discapacidad laboral provocada por dolor y la facilidad de expresión hasta obtener el nivel de saturación con una muestra de 5 trabajadoras. El nivel de saturación se obtuvo cuando dentro de las categorías en estudio no se encontraron datos nuevos emergentes, las categorías estuvieron ampliamente desarrolladas, demostrando variación y cuando se logró establecer una buena relación entre las categorías.

Como técnica de recolección de información se realizaron diez entrevistas en profundidad, dos a cada una de la participantes, teniendo en cuenta la disposición, el tiempo y el lugar elegido por las trabajadoras con una duración de promedio de una hora y 45 minutos cada una. Se utilizó una guía de entrevista previamente establecida. 
Todos los diálogos fueron grabados en audio previo consentimiento informado. Se transcribió el material de manera literal y se exportó el archivo al programa de análisis cualitativo de datos Atlas.ti (Muhr T. Scientific Software Development $\mathrm{GmbH}$, Berlín, Alemania), ingresando cada entrevista como una unidad hermenéutica. Se seleccionaron las citas que daban cuenta de cada uno de los componentes fenomenológicos y se les asignó un código con sentido, significado y estructura. Los códigos vinculados se agruparon en categorías que fueron relacionadas entre sí para crear redes semánticas que permitieron el análisis posterior 11. Los datos fueron obtenidos en julio de 2014 .

Se utilizó el método de análisis fenomenológico que consiste en escuchar detalladamente casos similares y describirlos con minuciosidad para elaborar una estructura representativa común de las experiencias vividas, tiene tres fases: descripción, reducción e interpretación. En la primera fase se utilizó el esquema de Patton 12 para describir las experiencias, comportamiento, opiniones, valores, sentimientos, sensaciones y conocimientos. En la segunda fase se determinaron los elementos fundamentales de la descripción, se codificaron y categorizaron según los cuatro existenciales básicos fenomenológicos: cuerpo, relaciones humanas, espacio y tiempo vividos. En la tercera etapa se especificó el sentido de la experiencia consciente de las mujeres trabajadoras con dolor crónico.

\section{Consideraciones éticas}

Todas las trabajadoras con dolor crónico osteomuscular que asistieron al servicio de ortopedia y neurocirugía del hospital y que cumplieron los criterios de selección, tuvieron la posibilidad de participar en el estudio sin ninguna discriminación. Se les explicó los objetivos de la investigación y el método de recolección de información. Su participación, así como la opción de retirarse en cualquier momento, fue voluntaria y se expresó de manera verbal. A las participantes se les garantizó el anonimato y la confidencialidad de la información, bajo los criterios éticos de la investigación con seres humanos de la Declaración de Helsinki y la Ley General de Salud de México, 1986 (Título II, Capítulo I, Art. 13, 16, 17), que califica esta investigación sin riesgo.

\section{Hallazgos}

De la población de 88 mujeres trabajadoras públicas del Estado de Jalisco que participaron en la investigación, 39,8\% realizó estudios superio- res, $50 \%$ reportó estar casada, $64,7 \%$ manifestó tener entre 1 y 3 hijos, 86,4\% afirmó que tenía a su cargo la realización de las labores domésticas además de su condición de trabajadora. En cuanto al cargo desempeñado, 34,1\% refirió ser docente y 26,1\% enfermera, el 46,6\% manifestó que asume sola la responsabilidad económica de sus hogares. La principal causa de dolor fue la artrosis de rodilla (17\%), seguida de hernia cervical (12,5\%) y lumbar (12,5\%). La cronicidad tuvo un rango de 6 meses a 31 años, más común entre 1 y 3 años (29\%); no se consideró para este estudio el origen laboral del dolor.

A partir de esta población, se entrevistó a 5 mujeres, con edades entre los 49 y 55 años de edad, todas con educación superior, dos se desempeñan como docentes, dos como enfermeras y una como abogada; todas aportan económicamente en sus familias, dos comparten los gastos $\mathrm{y}$ tres los asumen completamente. Han padecido dolor crónico en un periodo de dos a veinte años provocado por hernias cervicales y lumbares con radiculopatía, artrosis de rodilla, hallux valgus y ruptura del plexo braquial (Tabla 1).

En las entrevistas en profundidad se consideraron tres ejes temáticos. El primer eje: ser mujer abordó el significado de ser mujer, la experiencia dentro del rol familiar, social y laboral y las diferencias percibidas por género en cada uno de los roles. El segundo eje temático: calidad de vida laboral abordó el significado de trabajo, la experiencia de trabajar con un dolor crónico, tanto en la realización de las actividades cotidianas, como en las relaciones laborales, el apoyo recibido por las instituciones y el uso del tiempo libre. El tercer eje temático: dolor crónico osteomuscular indagó por el significado de dolor crónico, la experiencia de padecer un dolor crónico en el rol familiar, social y laboral y los cambios generados por la presencia de dolor. En la Tabla 2 se presentan algunas preguntas generadoras por cada uno de los ejes temáticos y de los descriptores, según el esquema de Patton, que formaron parte del guión de la entrevista.

\section{Fase 1: descripción fenomenológica}

Para la descripción de la información se utilizó el esquema propuesto por Patton quien considera las categorías: experiencia y comportamiento; opiniones y valores; sentimientos; sensaciones; y conocimiento.

\section{- Experiencias y comportamiento}

Cuatro de las cinco trabajadoras entrevistadas manifestaron que se han sentido discriminadas en el trabajo por motivo de su enfermedad; han 
Tabla 1

Datos sociodemográficos de las trabajadoras participantes en la entrevista en profundidad.

\begin{tabular}{|c|c|c|c|c|c|c|c|c|c|}
\hline Trabajadora & $\begin{array}{l}\text { Edad } \\
\text { (años) }\end{array}$ & Escolaridad & Estado civil & Hijos & $\begin{array}{c}\text { Doble } \\
\text { presencia }\end{array}$ & Cargo & $\begin{array}{l}\text { Responsabilidad } \\
\text { económica }\end{array}$ & $\begin{array}{l}\text { Años con } \\
\text { dolor }\end{array}$ & Diagnóstico \\
\hline Magnolia & 52 & Maestría & Soltera & 0 & Sí & Docente & Sola & 20 & $\begin{array}{c}\text { Hallux valgus y artrosis } \\
\text { de rodilla }\end{array}$ \\
\hline Iris & 54 & Técnica & Divorciada & 4 & Sí & Enfermera & Compartida & 3 & $\begin{array}{c}\text { Fibromialgia, sacroileitis } \\
\text { y radiculopatía }\end{array}$ \\
\hline Azalea & 49 & Licenciada & Soltera & 0 & Sí & Abogada & Sola & 2 & $\begin{array}{l}\text { Ruptura de bíceps } \\
\text { braquial, manguito } \\
\text { rotador y plexo braquial }\end{array}$ \\
\hline Margarita & 45 & Licenciada & Casada & 3 & No & Docente & Compartida & 6 & $\begin{array}{c}\text { Hernia cervical y lumbar } \\
\text { y radiculopatía }\end{array}$ \\
\hline Lirio & 55 & Licenciada & Soltera & 1 & Sí & Enfermera & Sola & 16 & $\begin{array}{c}\text { Hernia cervical y lumbar } \\
\text { y radiculopatía }\end{array}$ \\
\hline
\end{tabular}

Tabla 2

Preguntas orientadoras de las entrevistas en profundidad, según los ejes temáticos y los descriptores del esquema de Patton.

\begin{tabular}{|c|c|c|c|}
\hline \multirow{2}{*}{$\begin{array}{l}\text { Descriptores según } \\
\text { esquema de Patton }\end{array}$} & \multicolumn{3}{|c|}{ Ejes temáticos contemplados en la entrevista } \\
\hline & Ser mujer & Calidad de vida laboral & Dolor crónico osteomuscular \\
\hline $\begin{array}{l}\text { Experiencias y } \\
\text { comportamiento }\end{array}$ & $\begin{array}{c}\text { ¿Cómo es la experiencia de ser mujer } \\
\text { trabajadora? }\end{array}$ & $\begin{array}{c}\text { ¿Cómo es la experiencia de trabajar con } \\
\text { dolor crónico? }\end{array}$ & $\begin{array}{c}\text { ¿Cómo es la experiencia de padecer un } \\
\text { dolor crónico? }\end{array}$ \\
\hline Opiniones y valores & $\begin{array}{c}\text { ¿Qué representa para ti la familia y el } \\
\text { trabajo? }\end{array}$ & $\begin{array}{c}\text { ¿Cómo te ha apoyado la empresa ahora } \\
\text { que tienes dolor? }\end{array}$ & $\begin{array}{c}\text { ¿Qué representa para ti el padecimiento } \\
\text { del dolor? }\end{array}$ \\
\hline Sentimientos & $\begin{array}{c}\text { ¿Cómo te sientes con tu familia desde } \\
\text { que padeces dolor? }\end{array}$ & $\begin{array}{c}\text { ¿Cómo te sientes con tus compañeros } \\
\text { desde que estas enferma? }\end{array}$ & $\begin{array}{c}\text { ¿Qué sentimientos te genera sentir } \\
\text { dolor? }\end{array}$ \\
\hline Lo sensorial & $\begin{array}{c}\text { ¿Cómo es el trato en el trabajo cuando } \\
\text { una mujer padece dolor? }\end{array}$ & $\begin{array}{c}\text { ¿Cómo ha cambiado tu trabajo ahora que } \\
\text { tienes un dolor? }\end{array}$ & $\begin{array}{c}\text { ¿Qué dificultades has tenido en el trabajo } \\
\text { a causa del dolor? }\end{array}$ \\
\hline Conocimientos & & $\begin{array}{c}\text { ¿Sabes cómo tramitar tus restricciones } \\
\text { laborales? }\end{array}$ & $\begin{array}{c}\text { ¿Conoces cual es la causa y el pronóstico } \\
\text { de tu dolor? }\end{array}$ \\
\hline
\end{tabular}

sido víctimas de comentarios humillantes y de dudas con respecto de la verdadera naturaleza de su enfermedad, al insinuarles que puede ser una invención o un pretexto para obtener ganancias secundarias. Manifestaron no haber podido expresar su inconformidad por temor al despido o a represalias dentro del trabajo, además de tener que ejercer las tareas con exceso de esfuerzo, lo que generalmente redunda en mayor presencia de dolor. En su discurso utilizaron el término "falta de humanidad", refiriéndose no sólo al trato percibido por parte de los empleadores y compañeros de trabajo, sino también al trato recibido por los profesionales de la salud que les han prestado sus servicios. “...me sucedió la primera vez allí en medicina del trabajo, con un Dr. de apellido X que me dijo: 'Te vas a regresar a trabajar, ya no tienes derecho de volverte a incapacitar, porque te van empezar rebajar tu sueldo', y yo volteé y le dije a él: 'es que yo necesito mis dos extremidades para poder desempeñar mi trabajo'; y el me dijo: 'no, no, no, con una mano es suficiente para que realices tu trabajo..." (Azalea).

“...me era muy incómodo que me señalaran y me estuvieran diciendo: ¿Otra incapacidad? $\mathrm{Co}$ mo si hubiese sido gusto de uno, entonces yo lo sentía como marginación, este [...] como hostigamiento de alguna manera..." (Margarita). 
Todas manifestaron que la presencia del dolor les ha impactado negativamente su vida personal, familiar y laboral. Ahora tienen la necesidad de ser apoyadas en la realización de sus actividades de la vida diaria para continuar con sus rutinas. Esta dependencia les genera angustia y desesperación, pues perciben que incluso los integrantes de la familia se cansan y los compañeros de trabajo en muchos casos son forzados a auxiliarlas. Algunas de las participantes expresaron que guardan silencio e intentan esforzarse lo más que pueden para cumplir con todas las actividades asignadas, pero sienten gran frustración al ver que los resultados no son los esperados.

“...me siento limitada en muchos aspectos, el tener que depender de otras personas para poder hacer lo que yo antes hacía y que ya hoy no puedo hacer. Me siento limitada, desesperada..." (Azalea).

Por otro lado, las trabajadoras expresaron que, aunque se sientan con limitación para desempeñar sus actividades e incluso tengan una incapacidad laboral proferida por el médico, prefieren no tomar el tiempo, por temor a ser despedidas o por el impacto que la incapacidad tiene en la economía familiar, pues su salario disminuye un porcentaje según su duración.

“...cuando tú vas a una consulta médica, o bien te ponen una falta o te descuentan y eso es una realidad. Y muchas veces uno por esa situación uno se la piensa [...] porque aquí no es que tu vas a la consulta médica y está justificado, no [...] eso es una falta y a ti te descuentan..." (Magnolia).

\section{- Opiniones y valores}

Todas las participantes han tolerado una situación de inconformidad sentida por periodos superiores a dos años y en un caso hubo, además, hostigamiento y acoso laboral que ha tenido que ser expuesto ante las autoridades correspondientes. Las mujeres creen que las instituciones son permisivas con respecto a la indiferencia y apatía que se produce hacia ellas, tanto por parte de los directivos, jefes, compañeros y por las mismas instancias incluso legales, pues son muchas las trabas que encuentran para hacer uso de sus derechos.

“...[llorando] pues es una impotencia tremenda, falta de comprensión, falta de consideraciones, este... yo no me quiero sentir una persona discapacitada, limitada, no... pero si me gustaría tener un poquito, o recibir un poquito por parte de mis compañeros o de mis superiores, un trato humano y un trato consiente..." (Azalea).

“...Yo siento hostigamiento y eso [...] es una discriminación y no porque no pueda... o sea yo puedo hacer mi trabajo docente pero solamente con esas situaciones, que te dieran esa paz y esa tranquilidad que tú necesitas y el apoyo que necesitas como tal..." (Margarita).

En este sentido, es un hecho que las recomendaciones emitidas por el médico acerca de los cambios en las actividades laborales, así como modificaciones en el ambiente de trabajo no se ejecutan, lo que limita, retrasa e impide el desempeño cotidiano de la trabajadora enferma, provocando lo que ellas refieren como impotencia y desesperación.

“...de alguna manera actualmente con cierta frecuencia ocupan el laboratorio y pues tengo que estar subiendo y bajando escaleras a pesar de que allá saben que no lo puedo estar haciendo" (Margarita).

“...yo he visto con algunas compañeras que tienen problemas de columna, sobre todo las intendentes [...] es un trabajo pesado y les afecta el tener que mover los botes y a pesar de ello, no hay un cambio de actividad, que sería lo correcto ante un problema de salud..." (Magnolia).

Sin embargo, a pesar de las circunstancias se generan también mecanismos de resistencia y resiliencia, como respuesta para enfrentar su situación de dolor, los cuales están asociados con el alto grado de satisfacción que las mujeres sienten con su trabajo, su nivel de responsabilidad y la necesidad de mantenerse activas no sólo por el factor económico, sino por el hecho de demostrarse a sí mismas que son capaces de salir adelante y continuar.

“...trabajo es algo que dignifica, es algo que te hace sentir muy bien, tienes una recompensa, aunque no sea con dinero, es una recompensa interior de gozo, porque estás haciendo algo que te gusta..." (Iris).

“...Pues ahora sí que por la cultura que tenemos verdad... de que las mujeres tenemos que darnos y entregarnos con todo, no nos podemos rajar tan fácil [...] tengo que salir adelante no me puedo dar el lujo de quedarme sentada quejándome, tengo que luchar y tengo que luchar con todo, hasta donde definitivamente no pueda..." (Magnolia).

En sus relatos reconocen por su condición de género las diferencias culturales y el trato desigual, en cuanto a la discriminación y a la limitación para el ascenso laboral. Así mismo, refieren que dadas sus condiciones del rol tanto de madres, esposas o hijas, el dolor pasa a un segundo plano pues tienen múltiples tareas que resolver primero.

“...en nuestra cultura somos las mujeres muy resistentes, muy sumisas, con mucho valor al dolor [...] nos aguantamos el dolor y las mujeres tenemos el umbral al dolor más fuerte porque nos lo aguantamos, o sea no lo tenemos más fuerte, sino 
que aprendemos a que se haga algo secundario. Pasa a un segundo término" (Iris).

“...mi trabajo me gusta mucho, lo hago con gusto, lo único que no me satisface es que muchas de las veces el [...] vamos... el contexto también o con las personas con las que tú te relacionas en el trabajo, te ponen obstáculos y no te permiten hacer el trabajo, pues como realmente quisieras hacerlo. Yotra parte es la inestabilidad emocional que esto te genera por tener limitaciones en el mismo trabajo" (Margarita).

\section{- Sentimientos}

Los sentimientos encontrados fueron frustración, impotencia y percepción de injusticia. Las mujeres sienten que no son tratadas con consideración, sienten como si la gente quisiera ignorar que están enfermas, asignándoles las mismas tareas que cumplían antes y exigiéndoles el mismo cumplimiento. Soportan comentarios de compañeros y jefes en los que se insinúa, a veces de manera explícita, que se aprovechan de su condición para la extensión de las incapacidades, que no quieren trabajar, que inventan que están enfermas o que pareciera que no tienen nada. Sienten indiferencia por parte de las instituciones en cuanto al respeto de sus derechos como trabajadoras y como seres humanos.

“...pues la falta de humanidad y de conciencia, que no toda la gente te comprende que ya tienes una limitación, que no estás al 100\% y que ellos de cualquier manera quieren exigirte que tu sigas trabajando [...] hay mucha falta de comprensión, porque no entienden las limitaciones que tú tienes, para ellos lo primero es que salga el trabajo a costa de lo que sea..." (Azalea).

Se presenta también un sentimiento de tristeza que se manifiesta con llanto recurrente, asociado a la incapacidad de hacer cosas que antes se hacía o al no poder cumplir a cabalidad con todos sus roles, acompañado de sentimientos de desesperanza que aparecen a medida que el tiempo pasa y el dolor no mejora o la lesión resulta de difícil o imposible resolución. Para ellas, causa impotencia el sentir que se debe tomar muchos medicamentos diariamente que terminarán afectando la salud de otros órganos.

“...en un momento también me sentí desolada de no poder dar esa calidad y esa calidez... porque no me sentía bien yo misma, cuando tu no sientes eso, no puedes darlo, no puedes darlo a tu familia, tenía que yo curarme para empezar a florecer..." (Iris).

\section{- Lo sensorial}

Las trabajadoras perciben el dolor constantemente, refieren que es como un compañero que nunca las abandona, lo perciben como pesadez, adormecimiento y hormigueo que en algunas ocasiones se hace incluso evidente a los ojos de sus compañeros de trabajo.

“...después de traer mucho rato mi mano hacia abajo, empieza ese entumecimiento, ese hormigueo, ese cansancio..." (Azalea).

“...son medicamentos bastante fuertes les digo, si no quiero sentir dolor tengo que andar toda adormilada, para poder mitigar más mi dolor..." (Margarita).

\section{- Conocimiento}

Las trabajadoras han ido aprendiendo acerca de su enfermedad, saben cuáles son las causas, tratamientos, medicamentos y pronóstico esperado, el cual ven con pesimismo. Desconocen cuáles son sus derechos como trabajadoras, aunque han adquirido algunas nociones, no sólo por su propia experiencia, sino por la de los demás, a través de conversaciones en el servicio médico con otros trabajadores en su misma condición; dos de las entrevistadas refirieron que han aprendido y han tenido más claridad de los trámites que deben hacer tanto en el sindicato como en la atención médica, por recomendación de otros pacientes (Tabla 3).

“...entonces yo solicité las 12 horas que me faltaban para completar mi turno y brincaron mi derecho nuevamente, tuve que inconformarme y andar de un lado a otro y [...] el hecho es de que gané el juicio porque pues sí, efectivamente, era estar violando nuevamente mi derecho..." (Margarita).

\section{Fase 2: reducción fenomenológica}

A partir del análisis de la información, se crearon categorías y códigos, según los cuatro existenciales fenomenológicos (Tabla 4).

\section{- Relaciones humanas vividas}

En la familia, las relaciones se perciben con frustración provocada por la dependencia para la realización de actividades cotidianas, lo que conduce a que las mujeres callen su situación de enfermedad para no incomodar a otros. Esta situación es especialmente impactante en el caso de la convivencia de pareja, la cual se ve afectada por la dificultad que para algunas representa la intimidad y termina llevando con el tiempo al deterioro inevitable de la relación. 
Resumen de los hallazgos generales según el esquema de Patton.

\begin{tabular}{lc}
\hline Categorías & Hallazgos generales \\
\hline $\begin{array}{l}\text { Experiencia y comportamiento } \\
\text { Opiniones y valores }\end{array}$ & Discriminación, falta de consideración y humillación, segregación \\
$\begin{array}{l}\text { Sentimientos } \\
\text { Conocimientos }\end{array}$ & Frusticia, las instituciones son permisivas, no respeto por los derechos del trabajo, falta de humanidad impotencia, tristeza, desesperanza \\
Sensoriales & Derechos laborales, patología que se padece \\
\end{tabular}

Tabla 4

Categorías y códigos de los componentes fenomenológicos.

\begin{tabular}{|c|c|c|}
\hline Componente fenomenológico & Categoría & Códigos \\
\hline \multirow[t]{3}{*}{ Relaciones humanas vividas } & Familia & Apoyo; Silencio; Pareja; Dependencia; Rol \\
\hline & Trabajo & $\begin{array}{l}\text { Abuso de poder; Trato inhumano de compañeros y jefes; Falta de } \\
\text { credibilidad; Falta de apoyo; Estrés laboral; Recursos económicos } \\
\text { insuficientes; Sobrecarga laboral; Incumplimiento en la restricción } \\
\text { de tareas; Responsabilidad; Satisfacción por el trabajo }\end{array}$ \\
\hline & Personal de la salud & Abuso; Trato inhumano \\
\hline \multirow[t]{3}{*}{ Cuerpo vivido } & Subjetividad de los cambios corporales & $\begin{array}{l}\text { Rigidez; Adormecimiento; Cansancio; Pérdida de movimiento; } \\
\text { Alteración de una estructura corporal }\end{array}$ \\
\hline & Subjetividad de los cambios emocionales & Autoestima; Aceptación \\
\hline & Imaginario de las limitaciones & $\begin{array}{l}\text { Limitación en la función laboral; Limitación en la función vital; } \\
\text { Trabajo con dolor }\end{array}$ \\
\hline \multirow[t]{2}{*}{ Tiempo vivido } & Evocaciones & Pasado; Futuro \\
\hline & Tiempo en función del trabajo & Desgaste; Rumiación;Lentitud \\
\hline Espacio vivido & & En casa; En el trabajo \\
\hline
\end{tabular}

Las mujeres sienten que el dolor repercute en el desempeño de su rol como madres, esposas e hijas puesto que las limita para la realización de tareas domésticas que otros miembros deben asumir.

Las relaciones en el trabajo son percibidas como abusivas e irrespetuosas, desconsideradas frente a su discapacidad; reciben frecuentemente comentarios humillantes y discriminatorios, en algunos casos las tareas laborales se acumulan durante el tiempo de incapacidad y deben ser completadas después del reintegro. Debido a las normas legales vigentes, el salario disminuye después de cierto tiempo de incapacidad continua, lo que obliga a las mujeres a seguir trabajando a pesar de la presencia de dolor, para cumplir con sus responsabilidades económicas familiares. Esta situación genera angustia, desesperación y depresión que aumentan la sensación dolorosa y la sensación de sobrecarga laboral.
Se manifiesta una muy mala percepción en la atención de los profesionales de la salud a quienes describen como inhumanos e inconscientes de las dolencias ajenas. En general no solamente se sienten mal atendidas, sino también insuficientemente informadas con respecto de los procedimientos administrativos para el acceso eficiente a la atención médica.

“...en medicina del trabajo un Dr. X me dijo: 'Te vas a regresar a trabajar, ya no tienes derecho de volverte a incapacitar, porque te van empezar rebajar tu sueldo'; y yo volteé y le dije a él: 'es que yo necesito mis dos extremidades para poder desempeñar mi trabajo'; y él me dijo: 'no, no, no, con una mano es suficiente para que realices tu trabajo'..." (Azalea). 


\section{- Cuerpo vivido}

El cuerpo es vivido a partir de los cambios corporales y emocionales. Los principales cambios corporales percibidos son rigidez, adormecimiento, cansancio, pérdida de fuerza y movimiento experimentado como sensaciones molestas y discapacitantes que interrumpen e imposibilitan las actividades laborales y de la vida diaria.

Dentro de los cambios emocionales, las mujeres se sienten deprimidas e impotentes frente a su situación. Hay presencia de baja autoestima, expresada por el hecho de desplazar siempre la importancia de la propia salud por las responsabilidades, tanto familiares, como laborales. Cuatro de las mujeres participantes padecieron un dolor por largos periodos de tiempo sin consultar al médico, y manifestaron que acudieron sólo cuando ya no pudieron moverse, lo que evidentemente afecta el grado de cronicidad de la enfermedad.

\section{- Tiempo vivido}

El tiempo es percibido como evocaciones constantes de lo que antes se podía hacer y ahora no, y es vivido a través de la lentitud para el desempeño diario, generado por el dolor o el cansancio que aparecen más rápidamente. Ven el tiempo futuro con pesimismo; han aceptado su dolor porque no tienen otra alternativa, pero se sienten rendidas al pensar en los años que aún deben seguir laborando en las condiciones actuales, antes de poder acceder a una pensión de vejez, pues no contemplan en ningún caso la posibilidad de abandonar el trabajo por los compromisos económicos ya adquiridos.

\section{- Espacio vivido}

El espacio es vivido tanto en casa como en el trabajo a partir de las distancias, la fatiga que les provoca el desplazamiento y el desarrollo de actividades en movimiento por escaleras, calles y pasillos largos, además de la manipulación de peso, todo esto generador de gran inconformidad e incomodidad ante la presencia del dolor.

\section{Fase 3: interpretación fenomenológica}

En esta fase se intenta dar una interpretación de la experiencia de trabajar con dolor crónico osteomuscular, a partir de la descripción y reducción fenomenológica. Se encontró que el dolor es generador siempre de efectos negativos tanto en la familia, como en el trabajo. Los principales ejes de impacto familiar son la dependencia de otros miembros de la familia, la falta de recursos económicos a causa de las incapacidades largas y la frustración por las percepción de incompetencia en los roles de madre, esposa, hermana e hija, dentro de la mecánica familiar.

En el trabajo los ejes de impacto son la evidente limitación en la función laboral, que en algunos casos requiere apoyo de otros trabajadores o de una mayor inversión de tiempo para cumplirla, el trato indiferente frente a la discapacidad, la discriminación y la falta de confianza ante la presencia de la enfermedad. Se sienten indefensas al no poder manifestarse por temor a perder el empleo, porque perciben que las normas y sus derechos no son suficientes para que se les garantice un trato digno.

Esta situación les genera sentimientos de angustia, impotencia, tristeza, frustración e incomprensión que, combinado con el apoyo tanto de la familia y del medio laboral, determinan sus acciones de respuesta. Si el apoyo es positivo la respuesta será de aceptación del dolor crónico y una actitud positiva hacia el presente y el futuro a pesar de las circunstancias 13. Si el apoyo es negativo, la respuesta será de catastrofización y magnificación del dolor crónico, predecesores de depresión e incluso de aumento de dificultad en la resolución de las lesiones y resistencia al tratamiento 14 .

\section{Discusión}

La cotidianidad de la trabajadora con dolor crónico osteomuscular se ve marcada por la indiferencia, tanto de las instituciones para las que labora, como para los centros que ofrecen sus servicios de salud. Su principal experiencia está enmarcada con sentimientos de impotencia frustración e indefensión a los que se ven expuestas a causa de su enfermedad 15,16 y con poca posibilidad de actuación, debido al riesgo percibido de perder el empleo y la seguridad de un soporte económico para la familia. El medio laboral se presenta hostil y excluyente 17 , razón por la cual las trabajadoras ocultan en algunos casos la incapacidad emitida por el médico 18 .

El sistema legal del país en términos laborales no ofrece las garantías suficientes para un trabajo digno en condición de discapacidad a causa del dolor, puesto que las incapacidades frecuentes o prolongadas que se generan de una lesión del sistema osteomuscular disminuyen el salario 19 , obligando a las trabajadoras a mantenerse activas, aún cuando sus capacidades no sean suficientes.

Es de destacar que a pesar de las circunstancias adversas para la realización de las tareas, las mujeres sienten alto grado de responsabilidad y 
satisfacción personal por desempeñar su trabajo, lo cual, combinado con sus obligaciones económicas 20 , hace que cumplan con sus compromisos en cualquier condición laboral e inclusive desplazando su propia salud, lo que las predispone a una mayor carga familiar y social 21. Las mujeres que trabajan fuera del hogar asumen, además, el doble de responsabilidades que sus pares hombres 22 , generando gran dificultad en el desempeño de múltiples actividades 23 .

La falta de confianza y credibilidad relacionada con la presencia de la enfermedad puede estar asociada con la discriminación por género que cataloga a las mujeres como quejumbrosas y flojas para el trabajo, aunque esté comprobado que las mujeres presentan mayor morbilidad de dolor que los hombres 24,25.

Se encuentra como oportunidad, la posibilidad de aprovechar las condiciones laborales como una variable coadyuvante del manejo del dolor crónico osteomuscular, interviniendo las condiciones de ambiente y seguridad laboral, tanto en la adecuación de los espacios y procesos, como en el manejo de relaciones sociales al interior de la institución que permitan la sensibilización, el respeto, la inclusión y la no discriminación por el trabajo de un compañero enfermo.

Se encontró como barrera la mala imagen percibida de los profesionales de la salud en cuanto a la atención inhumana y desconsiderada 26,27,28; así mismo, la falta de oportunidad para el acceso a la atención médica especializada que cuenta con pocos profesionales adscritos, considerando la alta demanda de los servicios, lo cual alarga los tiempos de espera para la consulta incluso por meses.
Existe también falta de claridad en el manejo de una lesión de origen laboral y de las consecuentes incapacidades, en todos los actores, trabajadoras, empleadores, prestadores de los servicios de salud y sindicato, lo que prolonga los tiempos razonables para el reintegro, la reubicación o la calificación de la pérdida de capacidad laboral. Finalmente, es una barrera importante la falta de conocimiento de las mujeres frente a sus derechos como trabajadoras y sobre los procedimientos para hacerlos efectivos.

\section{Conclusiones}

La familia, como el trabajo, desempeñan un rol fundamental en cómo el dolor crónico osteomuscular es asumido por la trabajadora que lo padece. Su apoyo combinado con una eficiente prestación del servicio de salud y una normatividad que proteja a las trabajadoras enfermas son una necesidad sentida urgente de este grupo poblacional en el país.

La relación laboral podría utilizarse como una variable influyente en el manejo del dolor crónico osteomuscular, protegiendo la salud y ofreciendo posibilidades dignas de seguir realizando el trabajo lo que redundará en una mejor aceptación y por ende mejor calidad de vida.

Este estudio ratifica la necesidad de emprender investigaciones que reconozcan a las mujeres como sujetos ocupacionales diferentes, con particularidades sociales y psicológicas que las hacen más susceptibles y vulnerables de padecer dolor crónico osteomuscular. 


\section{Resumo}

O objetivo deste estudo foi conhecer as experiências de mulheres que trabalham com dor musculoesquelética crônica. O projeto foi qualitativo com abordagem fenomenológica. A técnica utilizada foi a entrevista em profundidade, realizada com cinco mulheres que trabalham com dor musculoesquelética superior a seis meses em ortopedia e serviços de neurocirurgia de um hospital em Guadalajara, México. Foram percebidas a rejeição, a segregação, a discriminação e a falta de apoio no seu ambiente de trabalho por causa de sua dor e sentimentos de frustração e impotência relacionados com o seu estado de saúde. São observadas como uma má percepção a barreira do pessoal de saúde e a falta de eficiência nos processos de deficiência, a reintegração e a localização do trabalho. As responsabilidades econômicas e familiares são a principal motivação para continuar a trabalhar com a presença de dor musculoesquelética crônica.

Dor Musculoesquelética; Dor Crônica; Trabalho

Feminino; Saúde da Mulher

\section{Colaboradores}

C. A. Ordóñez-Hernández contribuyó con la concepción del proyecto, análisis e interpretación de la información y redacción del artículo, y fue responsable de la exactitud e integridad de toda la obra. M. I. ContrerasEstrada contribuyó con la concepción del proyecto, revisión crítica relevante del contenido intelectual y aprobación de la versión final, y fue responsable de los aspectos del trabajo garantizando la exactitud e integridad de la obra. R. Soltero-Avelar contribuyó con la interpretación de los datos cualitativos, revisión crítica relevante del contenido intelectual y aprobación de la versión definitiva.

\section{Agradecimientos}

A los docentes tutores de esta investigación en la Escuela de Salud Pública de la Universidad de Guadalajara.

\section{Referencias}

1. Lasso C, Cruz A, Sánchez D, Toncel C. Dolor crónico en trabajadores de una empresa productora de comestibles. Ciencia \& Salud 2012; 1:45-9.

2. Araña M, Patten S. Trastornos musculo esqueléticos, psicopatología y dolor. Madrid: Secretaría de Estado para la Seguridad Social, Ministerio de Trabajo e Inmigración, Gobierno de España; 2011.

3. Zorrilla V. Trastornos músculo-esqueléticos de origen laboral en actividades mecánicas del sector de la construcción. Investigación mediante técnicas de observación directa, epidemiológicas y software de análisis biomecánico [Tesis de Doctorado]. Cáceres: Universidad de Extremadura; 2012.

4. Kaufman D. Neurología clínica para psiquiatras. Amsterdam: Elsevier Masson; 2008.

5. Husserl E. Investigaciones lógicas I. Madrid: Editorial Alianza; 1995
6. Berger P, Luckmann T. La construcción social de la realidad. Buenos Aires: Amorrortu Editores; 2001.

7. Ponty M. Fenomenología de la percepción. Barcelona: Planeta De Agostini; 1993.

8. Denzin N. Un punto de vista interpretativo. In: Denman C, Haro J, editores. Por los rincones. Hermosillo: El Colegio de Sonora; 2000. p. 147-205.

9. Yin R. Case study research. Design and methods. London: Sage; 1994.

10. Selltiz C. An introduction to sampling. In: Selltiz C, editor. Research methods in social relations. New York: Holt Rinehart; 1976. p. 516-25.

11. Martínez M. Uso del programa computacional Atlas.ti de Thomas Muhr (Univ. de Berlín) en la estructuración teórica de "datos" cualitativos. http:// prof.usb.ve/miguelm/estructuracionteorica\%203. html. 
12. Patton M. Qualitative research and evaluation methods. Thousand Oaks: Sage; 2002.

13. López A, Calero-García D. Sensibilidad al dolor, autorregulación, afrontamiento y personalidad en mujeres adultas sanas. Psicothema 2008; 20:420-6.

14. Fonseca N, Pereira F. Factores asociados a los disturbios músculo-esqueléticos en trabajadores de enfermería. Rev Latinoam Enferm 2010; 18:1-8.

15. Leite P, Merighi M, Silva A. Vivencia de una trabajadora de enfermería portadora de lesión "De Quervain”. Rev Latinoam Enferm 2007; 15:354-60.

16. Leite P, Silva A, Merighi M. A mulher trabalhadora de enfermagem e os distúrbios osteomusculares relacionados ao trabalho. Rev Esc Enferm USP 2006; 41:287-91.

17. Navarro A. ¿Mujeres proveedoras y jefas de familia? Nuevas realidades rurales en localidades de la región zamorana. La Ventana 2010; 31:139-71.

18. Knoche K, Sochert R, Houston K. La promoción de un trabajo saludable para los trabajadores con enfermedades crónicas: guía para una buena práctica. Leuven: European Network for Workplace Health Promotion/Health Programme of the European Union; 2012.

19. Instituto de Seguridad y Servicio Sociales de los Trabajadores del Estado. Ley del Instituto de Seguridad y Servicios Sociales de los Trabajadores del Estado. Diario Oficial de la Federación 2007; 31 mar.

20. Sánchez-Castillo C. Satisfacción de mujeres trabajadoras con la relación familia y trabajo. Rev Méd Inst Mex Seguro Soc 2012; 50:135-40.

21. Sotelo-Suarez N, Quiroz-Arcentales L, MahechaMontilla C, López-Sánchez P. Condiciones de salud y trabajo de las mujeres en la economía informal, Bogotá 2007. Rev Salud Pública 2012; 14: $32-42$.
22. Hoffmeister L, Vidal A. Desigualdades socioeconómicas en la salud percibida y declaración de dolor en población trabajadora chilena. Pers Soc 2012; 25:67-87.

23. Barbosa A, Pinto M, Fonseca S, De Oliveira D, Campos P. Ser docente de enfermería, mujer y madre: revelando la vivencia bajo la perspectiva de la fenomenología social. Rev Latinoam Enferm 2011; 19:1-8.

24. Rodríguez A. Aspectos epidemiológicos de las patologías más frecuentes asociadas al sexo femenino. Informe Médico 2009; 11:331-4.

25. Chacón F. Diferencias en la percepción del dolor entre hombres y mujeres. Papel de los estrógenos. Informe Médico 2009; 11:355-61.

26. Lavielle P, Clark P, Martínez H, Mercado F, Ryan G. Conducta del enfermo ante el dolor crónico. Salud Pública Méx 2008; 50:147-54.

27. Escudero-Carretero M, García-Toyos N, Prieto-Rodríguez M, Pérez-Corral O, March-Cerdá J, LópezDoblas M. Fibromialgia: percepción de pacientes sobre su enfermedad y el sistema de salud. Estudio de investigación cualitativa. Reumatol Clín (Barc) 2010; 6:16-23.

28. Martínez M. Experiencias y vivencias de mujeres con fibromialgia. Metas Enferm 2009; 12:26-30.

Recibido el 25/Nov/2014

Versión final presentada el 09/Abr/2015

Aprobado el 04/May/2015 